\title{
Mucormicosis «rinoorbitaria» en niños con inmunosupresión. Serie de tres casos y revisión de la literatura
}

\author{
«Rhinoorbital» mucormycosis in children with immunosuppression. \\ Series of 3 cases and review of the literature
}

\author{
Gabriela Estefanía Valdés de la Torre,* María Elena Martínez Bustamante, \\ Alfredo Raúl Rodolfo Morayta Ramírez Corona ${ }^{\S}$ \\ * Residente de $5^{\circ}$ grado de Infectología Pediátrica. \\ ${ }^{\ddagger}$ Adscrito al Servicio de Infectología Pediátrica. \\ $\S$ Jefe del Servicio de Infectología Pediátrica. \\ Servicio de Infectología Pediátrica, Coordinación de Pediatría. Centro Médico Nacional 20 de Noviembre del Instituto \\ de Seguridad y Servicios Sociales de los Trabajadores del Estado (ISSSTE). Ciudad de México.
}

\section{RESUMEN}

La mucormicosis es una infección por hongos oportunistas del orden mucorales y tercera causa de infección fúngica invasiva, es de baja incidencia, pero alta mortalidad (50-90\%), generalmente se manifiesta en huéspedes inmunocomprometidos. Se adquiere por inhalación, ingestión y/o contaminación de heridas de esporangiosporas que se encuentran en el medio ambiente. La principal presentación clínica en niños es la rinoorbitocerebral. El pilar fundamental en el pronóstico es la sospecha, el inicio de terapia fúngica anticipada y el desbridamiento del tejido necrótico. Presentamos serie de tres casos de niños con enfermedad hematológica e inmunosupresión secundaria con mucormicosis, forma diagnóstica, tratamiento y desenlace.

Palabras clave: Mucormicosis, inmunosupresión, anfotericina B.

\section{INTRODUCCIÓN}

La mucormicosis (previamente zigomicosis) es una enfermedad rara ocasionada por hongos oportunistas, saprófitos, aeróbicos, del orden mucorales, familia Mucoraceae. Se presenta con predominio

Financiamiento: Ninguno.

Conflicto de intereses: Ninguno.

Derecho a la privacidad y consentimiento

informado: Los autores declaran que en este

artículo no aparecen datos personales del paciente.

https://dx.doi.org/10.35366/92386

Rev Latin Infect Pediatr 2020; 33 (1): 49-56

\section{ABSTRACT}

Mucormycosis is an infection by opportunistic fungi of the order Mucorales and third cause of Invasive Fungal Infection, is of low incidence, but high mortality (50-90\%), usually occurs in immunocompromised hosts. It is acquired by inhalation, ingestion and / or contamination of wounds by sporangioespore, which are found in the environment. The main clinical presentation in children is rhino-orbitocerebral. The fundamental pillar in the prognosis is the clinical suspicion, early fungal therapy and debridement of the necrotic tissue. We present a series of 3 cases of children with haematological disease and secondary immunosuppression with mucormycosis, diagnostic method, treatment and outcome.

Keywords: Mucormycosis, immunosuppression, amphotericin B.

en pacientes inmunocomprometidos, asociada a una alta mortalidad por el tropismo hacia los vasos sanguíneos y rápida progresión. ${ }^{1,2} \mathrm{La}$ incidencia anual a nivel mundial es de 0.4-1.7 casos por cada $1,000,000$ habitantes, en pacientes con leucemia la incidencia es de ocho casos. ${ }^{3}$

Los géneros que habitualmente infectan al hombre dependen del área geográfica, en Norteamérica el más común es Rhizopus spp. (52\%), seguido de Mucor spp. (23\%), Rhizomucor spp. (7\%), Lichtheimia spp. (3\%), Cunninghamella, Apophysomycesy Saksenaea. En el estudio francés RetroZygo, el más común fue Rhizopus arrhizus (oryzae) (32\%), Lichtheimia spp.
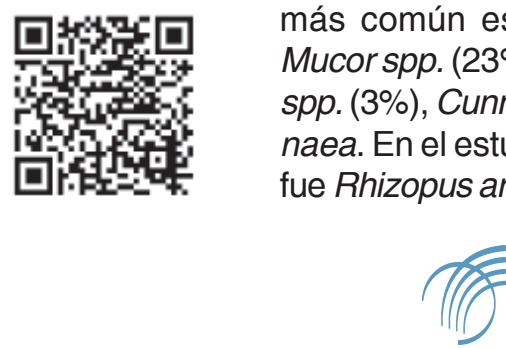
(29\%), Rhizopus microsporus (17\%). En un registro en la India el género más aislado fue $R$. arrhizus (69\%), seguido de Apophysomyces elegans (19\%). 4,5

Se encuentran distribuidos en el medio ambiente, suelo, materia orgánica en descomposición, excreta animal y aire. ${ }^{6}$ Se adquieren principalmente por inhalación de esporas, inoculación de heridas o por ingestión de esporas. Presentan tropismo por los vasos sanguíneos, generando angioinvasión y una rápida progresión. Las esporas inhaladas germinan en hifas dentro del endotelio vascular produciendo trombosis arteriovenosa. ${ }^{4,7,8} \mathrm{La}$ mucormicosis está asociada con pacientes inmunocomprometidos, en los inmunocompetentes es colonizante del aparato respiratorio y gastrointestinal. Los principales factores de riesgo en niños son prematurez, neutropenia profunda y prologada, neoplasia hematológica, trasplante de células hematopoyéticas u órgano sólido, inmunodeficiencias, tratamiento con inmunosupresores, trauma previo y quemaduras. ${ }^{2,9}$

Las principales formas de presentación clínica son la rinoorbitocerebral (50\%), pulmonar (30\%), gastrointestinal, cutánea y diseminada. ${ }^{2}$ En niños, la forma rinoorbitocerebral es la más frecuente $(77 \%)$.

La sospecha diagnóstica es clínica tomando en cuenta los factores de riesgo que presenta, inicia con edema periorbital, dolor facial, alteraciones visuales, lesiones necróticas en paladar o tabique nasal, rinorrea mucosanguinolenta y fiebre. , $^{1,2,4}$

El diagnóstico se realiza con examen directo microscópico, cultivo del tejido y estudio histopatológico. Para el control de la infección y mejorar el pronóstico, el tratamiento debe ser inmediato, multidisciplinario, terapia antifúngica y desbridamiento quirúrgico extenso. ${ }^{8}$

La epidemiología en niños está muy limitada, es difícil de estimar por ser una infección poco común y difícil de diagnosticar. ${ }^{9}$ Se realizó un estudio epidemiológico en países europeos y no europeos donde se registraron todos los casos confirmados de mucormicosis invasiva en niños entre los años de 2005 a 2014 se registraron 63 casos, 54 (85.7\%) eran europeos y nueve casos no europeos, la mayoría presentaba una neoplasia hematológica de base (46\%). Se registró una mortalidad global de $33.3 \%$, $61 \%$ fue mucormicosis diseminada, de 41 a $66 \%$ tenía neoplasia de base y $16.6 \%$ en pacientes sanos. ${ }^{9}$ En este estudio no se pudo sacar la incidencia de mucormicosis en niños, ya que fueron pocos casos registrados.

En México los casos son esporádicos y hay pocos reportes de casos. En el Instituto Nacional de
Pediatría informaron durante el periodo 1998-2010 una incidencia de $4 \%$ de todas las micosis de vías aéreas. ${ }^{2}$ El Hospital General de México reportó en un estudio retrospectivo de 1985 a 2012, 168 casos de mucormicosis, de los que $13.9 \%$ correspondían a niños. ${ }^{2}$

Aunque la infección tiene una elevada mortalidad, el pronóstico en general ha mejorado en el transcurso de los últimos años debido a la aplicación de medidas terapéuticas implementadas de manera precoz y oportuna. ${ }^{4}$

Presentamos una serie de tres casos de mucormicosis en pacientes pediátricos inmunocomprometidos en un hospital de tercer nivel en México.

\section{PRESENTACIÓN DE CASOS}

\section{Caso 1}

Paciente femenino de nueve años de edad, previamente sana, antecedente de dos meses de evolución con fiebre y petequias. Ingresa con fiebre sin foco aislado, laboratorios con pancitopenia, confirmando diagnóstico de anemia aplásica. Inicia tratamiento con tiroglobulina y esteroides sin mejoría. Una semana después tiene nuevo evento de fiebre complicándose con choque séptico por Klebsiella pneumoniae y colitis neutropénica, completando 14 días con carbapenémico. Durante su estancia presenta aumento de volumen palpebral derecho (clasificándolo como día 1 por manifestaciones clínicas), valorada por el servicio de oftalmología, quienes reportan diagnóstico de celulitis preseptal (Figura 1). Día +5 : se observa progresión cutánea con región eritematosa, edema centrofacial invo-

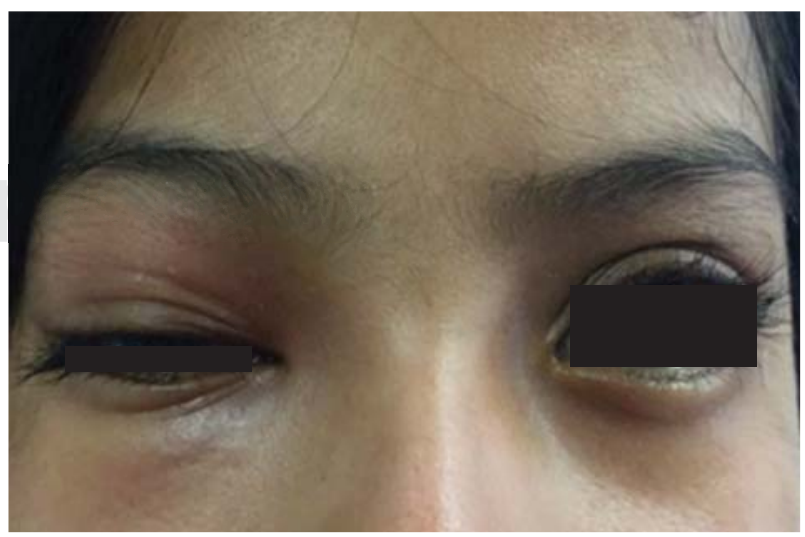

Figura 1: Celulitis preseptal (día +1). 


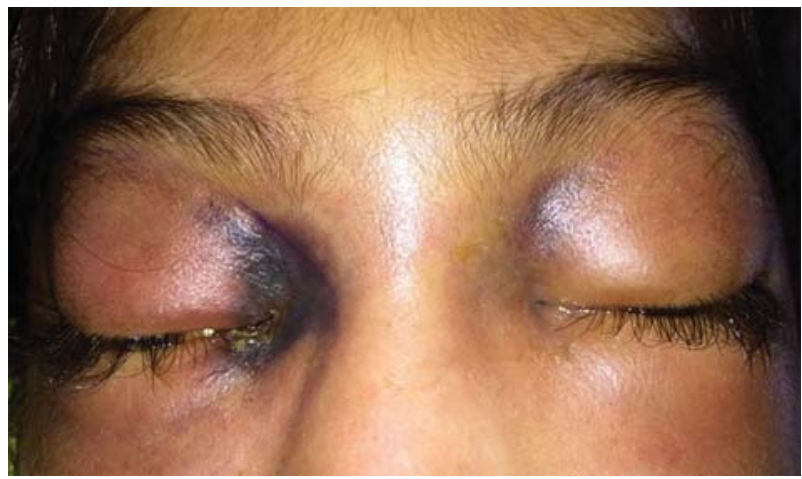

Figura 2: Progresión cutánea, edema bipalpebral y centrofacial con zonas necróticas (día +5$)$.

lucrando dorso nasal y dos tercios mediales de párpados inferiores y superiores, áreas necróticas cantales internas y secreción negruzca escasa a través de las mismas (Figura 2), se realiza TAC observando ocupación de celdillas etmoidales, seno esfenoidal y maxilares. Ante sospecha clínica de mucormicosis, se inicia anfotericina $\mathrm{B}$ lipídica a 5 $\mathrm{mg} / \mathrm{kg} / \mathrm{día}$, solicitando de manera urgente desbridación quirúrgica. La paciente no se encontraba en condiciones para realizar tratamiento quirúrgico, por necesidad de retraso se decide agregar doble esquema antifúngico con caspofungina al no contar con posaconazol. Día +7: se efectúa desbridación quirúrgica por parte del servicio de otorrinolaringología y oftalmología, obteniendo tejido necrótico en techo de fosa nasal, cornetes medios y septum, seno esfenoidal, etmoidal y maxilar; tejido necró- tico en región periocular bilateral con involucro de ambos párpados y vía lagrimal, áreas de necrosis sobre globos oculares, los cuales son preservados e irrigados con anfotericina. Examen directo con presencia de hifas hialinas, cenocíticas, macrosifonadas (Figura 3). Día +9: extensión de áreas de necrosis, se realiza segunda desbridación y aplicación de anfotericina perilesional. Día +11 : progresión de lesión en macizo facial con áreas extensas de necrosis (Figura 4). Día +13: TAC con desorganización de globos oculares, senos maxilares y etmoidales ocupados. Día +18: defunción por choque séptico y progresión de mucormicosis rinoorbitaria. Cultivo con identificación Mucor ramosissimus.

\section{Caso 2}

Paciente masculino de 15 años con antecedente de un mes previo de diagnóstico de leucemia linfoblástica aguda B. Cursa día +25 de inducción a la remisión. Presenta lesión necrótica en mucosa de cornete medio derecho, aumento de volumen en dorso nasal y lesión violácea paladar duro. Día +1: TAC de senos paranasales con pansinusitis y sospecha diagnóstica de mucormicosis, se inicia tratamiento con anfotericina B lipídica. Día +3: se realiza cirugía endoscópica de nariz y senos paranasales, mucosa necrótica de pared lateral nasal, cornete medio e inferior, techo seno maxilar y dehiscencia de pared posterior derecha, en la exploración oftalmológica se descarta invasión orbitaria. Examen directo con hifas hialinas

\section{Figura 3:}

Tinción azul de lactofenol, hifas hialinas, no septadas.
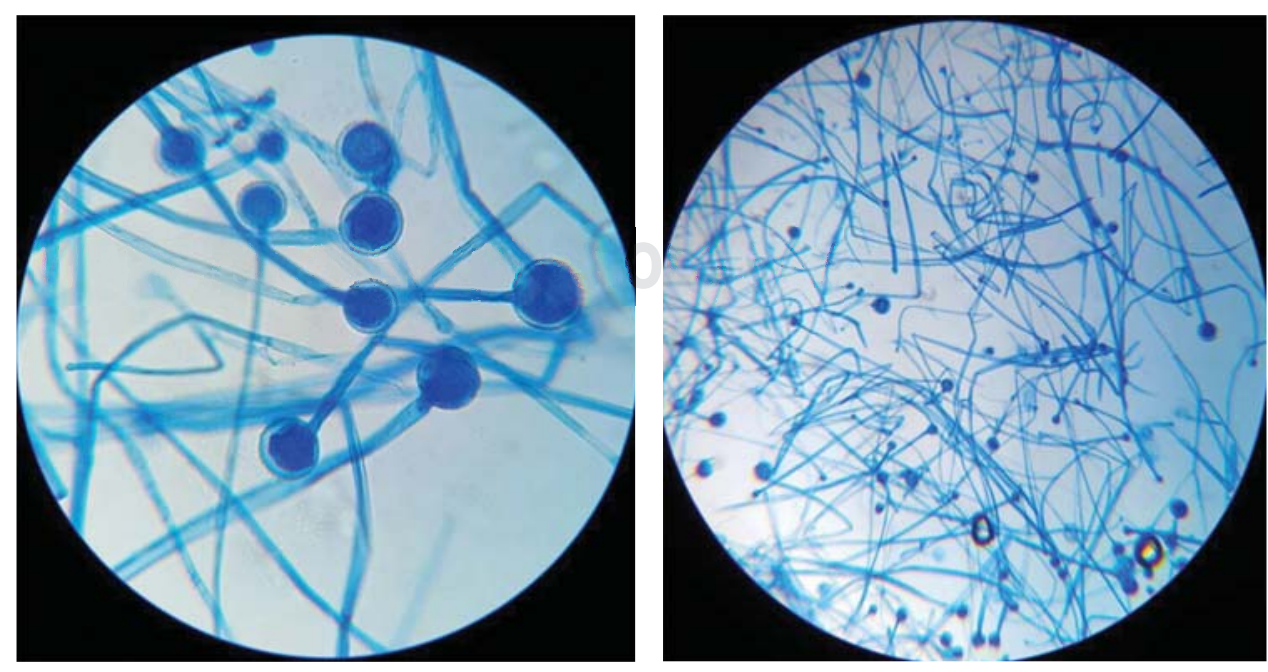
macrosifonadas, no septadas. Día +6: segunda cirugía endoscópica y desbridamiento dorso nasal. Día +8: reporte final de cultivo con identificación de Rhizopus oryzae (arrhizus), agregándose a tratamiento caspofungina. A pesar de tratamiento quirúrgico persisten bordes necróticos, se colocan lavados nasales con anfotericina B. Continua febril y con neutropenia prolongada, complicándose con absceso perianal de $4.3 \mathrm{~cm}^{3}$, completa esquema con meropenem y vancomicina por 14 días. Día +9 : tercer desbridamiento quirúrgico de nariz y senos paranasales, bordes necróticos en dorso nasal, rama ascendente del maxilar y huesos propios de la nariz, piso nasal desde el cuerpo de cornete hasta nivel de coana necrótico. Día +12: se agrega involucro orbitario derecho. Día +13 : desbridamiento quirúrgico en dorso nasal y región centrofacial, por parte de oftalmología se observan cambios en la coloración de lámina cribosa del etmoides, se realiza legrado de pared lateral y superior de antro maxilar, retirando lámina papirácea. Día +20: diagnóstico anatomopatológico de biopsia de pared media y órbita con necrosis e hifas. Día +27: biopsia cultivo de heridas negativo para hifas. Día +39 : biopsia cultivo de costras y mucosas positiva para hifas hialinas. Día +42: desbridamiento quirúrgico órbita derecha, se retira piso de órbita, $50 \%$ del piso maxilar y biopsia de hueso. Día +54 : examen directo positivo con visualización de hifas macrosifonadas, cenocíticas, hialinas. Continúan bordes de heridas con necrosis e hifas, con necesidad de múltiples intervenciones quirúrgicas hasta presentar bordes libres de infección. Por indicación familiar deciden no continuar con tratamiento. Día +61 : resultado

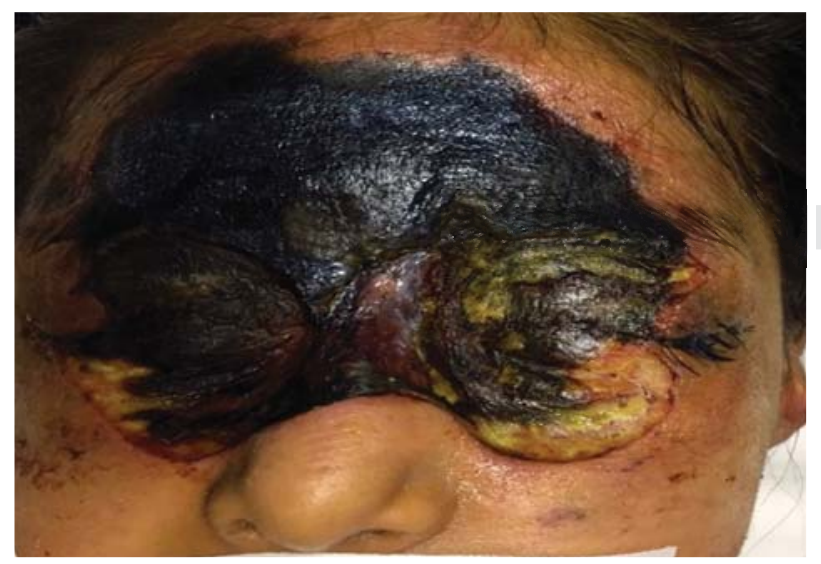

Figura 4: Necrosis centrofacial con afectación de globos oculares (día +11).

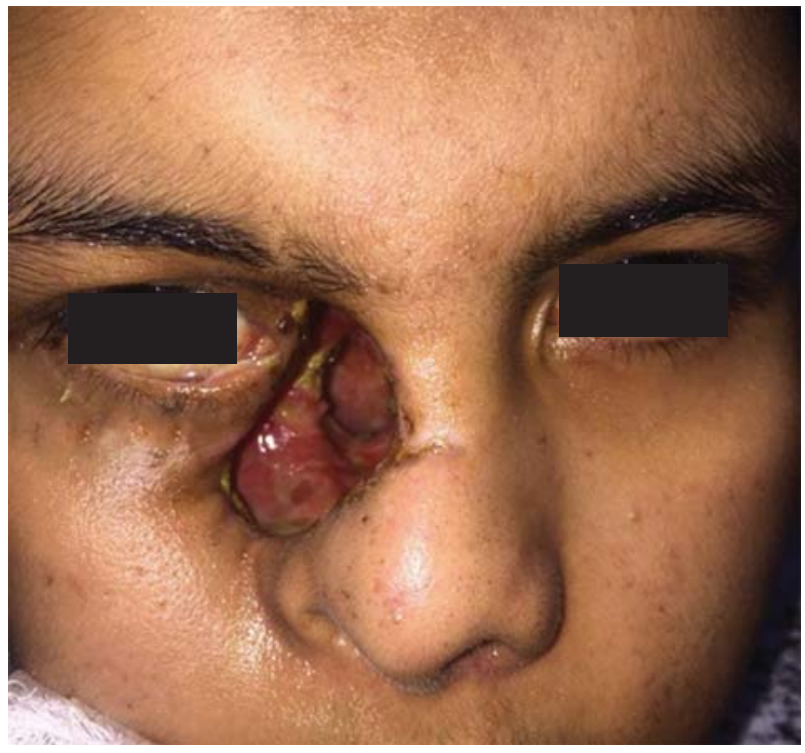

Figura 5: Región necrótica en receso frontoetmoidal y techo etmoidal. Seguimiento día +103 .

anatomopatológico de senos paranasales y hueso de seno maxilar con tejido necrótico y presencia de hifas. Día +75: egreso hospitalario por máximo beneficio, completando 75 días de anfotericina B y 67 con caspofungina. Seguimiento por consulta externa de oftalmología, otorrinolaringología e infectología a los 28 días (día +103) con persistencia de región necrótica hacia receso frontoetmoidal, techo etmoidal y piso orbitario derecho sin evidencia de lesión (Figura 5). Día +151: ingresa a urgencias por choque séptico. Día +152: defunción.

\section{Caso 3}

Paciente femenino de 16 años con antecedente de leucemia linfoblástica aguda $b$, de dos años de evolución, actualmente con recaída temprana a médula ósea, en quimioterapia de citorreducción. Presenta fiebre y neutropenia de alto riesgo completando tratamiento con cefalosporina de cuarta generación por siete días. Tres días después inicia con lesión violácea en paladar duro (Figura 6) y lesión necrótica en base de narina derecha (día +1$)$, sospecha de mucormicosis, se inicia tratamiento con anfotericina B lipídica a $5 \mathrm{mg} / \mathrm{kg} / \mathrm{día}$, valorada ese mismo día por otorrinolaringología detectando lesión necrótica en fosa nasal derecha de $1 \mathrm{~cm}$, cornete medio y paladar duro con lesión violácea de $1 \mathrm{~cm}$. Examen directo con hifas, hiali- 


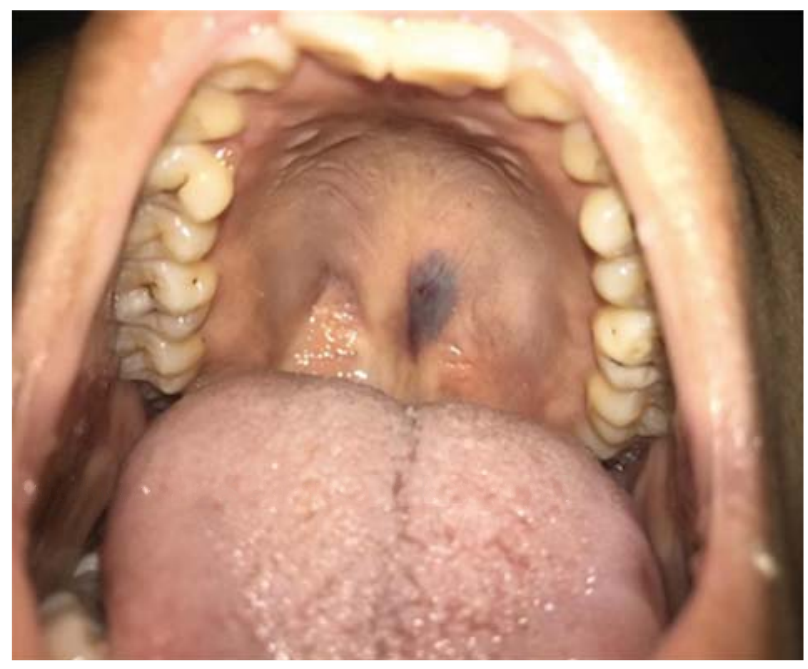

Figura 6: Lesión violácea paladar duro (día +1).

nas, no septadas, indicación de desbridamiento quirúrgico de urgencia, los familiares rechazan consentimiento de cirugía por los cambios estéticos que sufriría la paciente, se informa a los padres la necesidad del tratamiento quirúrgico para control de la infección y disminuir el riesgo de mortalidad. Día +3: los padres aceptan procedimiento quirúrgico, realizándose desbridamiento de costra necrótica en fosa nasal y piso nasal derecho, maxilofacial reseca lesión necrótica de paladar duro. Día +7 : se mantuvo en vigilancia estrecha sin presentar datos de progresión, con bordes libres (Figura 7). Día +10: se recaba reporte anatomopatológico con hifas y tejido necrótico. Día +11 : inicia con sangrado de tubo digestivo bajo. Día +12: defunción por choque hipovolémico.

\section{DISCUSIÓN}

El diagnóstico de mucormicosis sigue siendo un reto, pacientes con factores de riesgo y sospecha clínica deberán iniciar tratamiento de manera inmediata para tratar de controlar la angioinvasión y disminuir tasa de mortalidad.

La presentación clínica de los casos descritos es la más común en pacientes pediátricos, la mala evolución que mostraron fue principalmente por complicaciones de la enfermedad de base, sólo el primer caso fue por la demora en el diagnóstico e inicio tardío del tratamiento. El tratamiento inicial fue con anfotericina B liposomal como tratamiento de elección para mucormicosis, en el caso 2 se agrega doble esquema con caspofungina por aislamiento de $R$. oryzae, sensible a equinocandinas. En nuestra institución no se cuenta con posaconazol como tratamiento de salvamento.

La sospecha diagnóstica inicia con la clínica y sí muestra factores de riesgo como prematurez, neutropenia profunda y prologada, neoplasia hematológica, postrasplantados de células hematopoyéticas, trauma previo, corticoides sistémicos, inmunosupresión severa, profilaxis con voriconazol., ${ }^{70}$ Los síntomas iniciales en la presentación clínica rinoorbital son edema periorbitario, alteraciones en la visión, parálisis facial, dolor facial, lesiones violáceas o necróticas en narinas o paladar, rinorrea mucosanguinolenta y fiebre. ${ }^{10,11}$

Existen múltiples métodos para apoyar el diagnóstico, el cultivo de tejidos es el de elección. ${ }^{9}$ La Confederación Europea de Micología Médica (ECMM) y la Sociedad Europea de Microbiología Clínica y de Enfermedades Infecciosas (ESCMID) recomienda el examen directo con tinción de calcoflúor para la detección de hifas de mucorales, las cuales son largas de 6-25 $\mathrm{mcm}$ (micrometros), no septadas, hialinas, con ángulo $90^{\circ}$. Posterior a la identificación microscópica, se debe realizar cultivo para confirmar el diagnóstico, identificación de la especie y susceptibilidad antifúngica. El estudio histopatológico puede dar un diagnóstico sugestivo si las hifas son características, acompañado de datos de necrosis y angioinvasión. . $^{511}$

Como pruebas séricas los galactomananos y $\beta$-glucanos utilizados para el diagnóstico de enfermedad fúngica invasiva son negativos en los

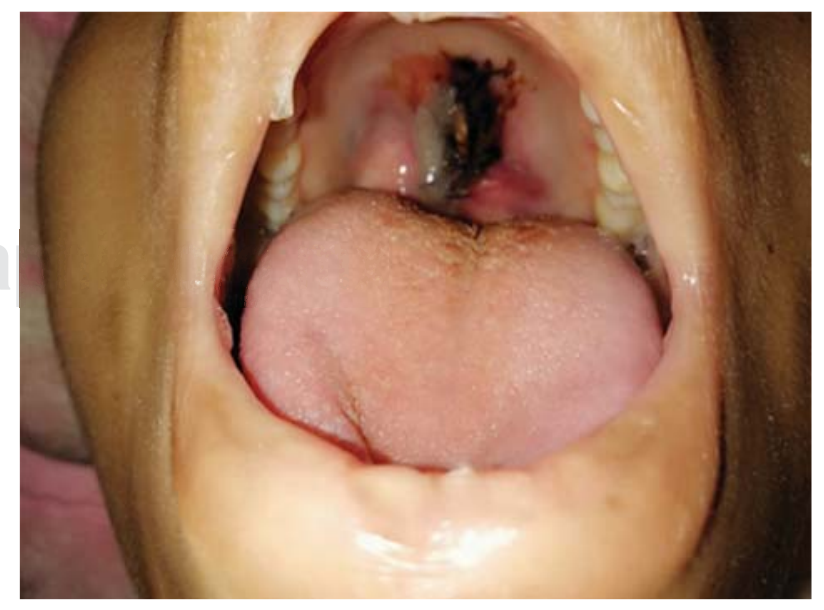

Figura 7: Sin datos de progresión con bordes libres (día +7). 
mucorales, sólo son útiles para descartar diagnósticos diferenciales como Aspergillus. ${ }^{9}$ A pesar de que son angioinvasivos, los hemocultivos raramente son positivos. $^{12}$

Hasta ahora ninguno de los biomarcadores fúngicos son útiles para el diagnóstico de mucormicosis. La detección de ADN o antígenos aún no está estandarizada. ${ }^{13}$

El estudio de imagen de elección es la tomografía axial computarizada (TAC) con contraste donde se observan opacificaciones en senos paranasales, edema de mucosa, edema de tejidos blandos y músculos extraoculares, así como destrucción ósea. Se solicita resonancia magnética cuando se presenta infección intracraneal e invasión vascular. ${ }^{3}$

El tratamiento debe iniciarse de manera inmediata ante la sospecha clínica para disminuir el riesgo de mortalidad. El tratamiento actual utilizado es el desbridamiento quirúrgico extenso del tejido necrótico y terapia antifúngica sistémica. ${ }^{3,14-16}$ Para un control local de la infección es necesaria la resección de todo el tejido necrótico, donde la penetración del antifúngico es baja, normalmente son necesarias varias intervenciones hasta lograr áreas libres de enfermedad. El tratamiento quirúrgico disminuye el riesgo de muerte en $79 \%$., 16,17

Como tratamiento de primera línea para mucormicosis autorizado por la FDA (United States Food and Drug Administration) es la anfotericina B liposomal a dosis de $5-7 \mathrm{mg} / \mathrm{kg} / \mathrm{di}$. En estudios in vitro muestra excelente actividad vs. mucorales. ${ }^{5}$

Como tratamiento de segunda línea por resistencia a anfotericina, alergia o como terapia de salvamento está el posaconazol como terapia combinada. Presenta actividad in vitro e in vivo versus mucorales, entre 64 y $100 \%$ de las cepas tienen MICs $<0.5 \mu \mathrm{g} /$ $\mathrm{mL}$ a posaconazol. Es utilizado como tratamiento de salvamento combinado con anfotericina $B$, con buena respuesta entre 62 y $79 \%$ de supervivencia o terapia de mantenimiento cuando hay una resolución completa en estudio de imagen y corrección de factores de riesgo. La absorción de posaconazol vía oral es alta, se modifica con los alimentos, mucositis, $\mathrm{pH}$ gástrico y motilidad. La ECMM/ESCMID lo recomienda altamente como terapia de salvamento, la duración no está establecida. ${ }^{5,15}$ No existen estudios donde se recomiende el uso de posaconazol como monoterapia o de primera línea.

Respecto a otros azoles, itraconazol tiene actividad limitada, mientras que voriconazol y fluconazol no tienen actividad contra mucorales. ${ }^{17-21}$
Las equinocandinas (caspofungina, anidulafungina, micafungina) no tienen eficacia contra mucorales; sin embargo, Rhizopus oryzae presenta un blanco específico para equinocandinas, las cuales se utilizan como tratamiento de salvamento junto con anfotericina $B{ }^{22}$

En estudios in vitro se ha demostrado la ausencia de antagonismo entre anfotericina $B$ y posaconazol, la combinación de ambos fármacos hace sinergia $40 \%$ contra hifas y $10 \%$ vs. conidias. ${ }^{19,22}$

El deferasirox en un quelante de hierro que suprime la proliferación de mucorales al deprivarlos de hierro, no se recomienda su uso como tratamiento, ya que no se ha comprobado su eficacia. ${ }^{12}$

La duración del tratamiento antifúngico se determina de forma individualizada dependiendo la evolución clínica, pero se deberá llevar al menos por seis a ocho semanas. ${ }^{5}$ ECMM/ESCMID recomienda continuar con terapia antifúngica hasta resolución completa en estudio de imagen y corrección de factores de riesgo, en ese momento se puede iniciar una terapia de mantenimiento con posaconazol. En caso de que no exista una resolución, se puede aumentar la dosis de anfotericina B (7-10 mg/kg/8 día). 5,12,14,15,17

Chamilos y colaboradores reportaron dos veces más riesgo de mortalidad en el retraso del inicio del tratamiento (seis días después del diagnóstico) comparado con el inicio inmediato ( 82.9 vs. $48.6 \%$ ). ${ }^{12}$

En las guías del uso de antifúngicos en pacientes con enfermedad fúngica invasiva de Taiwán de 2016, el tratamiento recomendado para mucormicosis es anfotericina B liposomal, y como terapia de salvamento o de mantenimiento posaconazol, junto con la corrección de las condiciones predisponentes que presente el paciente como neutropenia y disminución de inmunosupresores. ${ }^{1}$

Para prevenir la infección por mucorales es importante tener un control ambiental, evitar estar en contacto o cerca de construcciones, por la suspensión de esporas en el aire y el riesgo de inhalación de las mismas. La profilaxis antifúngica para Candida y Aspergillus está indica en pacientes inmunocomprometidos con alto riesgo de infección fúngica invasiva; sin embargo, no actúa contra mucorales. En los nuevos estudios se sugiere posaconazol como fármaco de elección para profilaxis antifúngica en pacientes postrasplantados, teniendo así una cobertura para mucorales. Se realizaron dos estudios prospectivos comparando posaconazol con fluconazol e itraconazol como profilaxis en postrasplantados. En el grupo con fluconazol e itraconazol se reportó un caso de mucormicosis, en comparación con el 
Tabla 1: Comparación de la actividad de anfotericina B, posaconazol e itraconazol contra 10 mucorales en 216 casos clínicos.

\begin{tabular}{lccc} 
& $\begin{array}{c}\text { Anfotericina B } \% \text { con } \\
\text { MIC } \leq 1 \mu \mathrm{g} / \mathrm{mL}\end{array}$ & $\begin{array}{c}\text { Posaconazol \% con } \\
\text { MIC } \leq 5 \mu \mathrm{g} / \mathrm{mL}\end{array}$ & $\begin{array}{c}\text { Itraconazol \% con } \\
\mathrm{MIC} \leq 5 \mu \mathrm{g} / \mathrm{mL}\end{array}$ \\
\hline Rhizopus sp. (101) & 100 & 80 & 62 \\
Rhizopus arrhizus (20) & 100 & 64 & 50 \\
Rhizopus microsporus (12) & 100 & 78 & 60 \\
Mucor sp. (41) & 94 & 70 & 57 \\
Mucor circinelloides (6) & 100 & 0 & 0 \\
Rhizomucor sp. (5) & 100 & 67 & 67 \\
Lichtheimia sp. (3) & 100 & 100 & 50 \\
Lichtheimia corymbifera (9) & 100 & 100 & 100 \\
Cunninghamella sp. (13) & 63 & 75 & 29 \\
Apophysomyces elegans (6) & 100 & 83 & 80
\end{tabular}

grupo con posaconazol, en el cual no se presentaron casos (Tabla 1). ${ }^{12,18,21}$

La mortalidad depende de la enfermedad de base que muestre el paciente, sitio de infección, especie fúngica y tratamiento establecido. Como factores de mal pronóstico se encuentran pacientes con neoplasia y neutropenia, uso previo de antifúngicos como caspofungina o voriconazol y la detección de la especie Cunninghamella spp. ${ }^{12}$

En un estudio epidemiológico realizado en Europa, siendo el análisis multinacional más grande de casos de mucormicosis en edad pediátrica, se concluye que el tratamiento quirúrgico abrasivo y el inicio de antifúngico sistémico de inicio temprano tiene una mortalidad de $18.5 \%$, con tratamiento antifúngico sistémico sin desbridamiento quirúrgico es de $60 \%$ y sin tratamiento es de $100 \% .^{9}$

En un estudio multicéntrico prospectivo de pacientes postrasplantados de células hematopoyéticas de la red de vigilancia de infecciones asociadas a trasplantes (TRANSNET) entre los años 2001 y 2006 reporta que la frecuencia de mucormicosis es la tercera causa de infección $8 \%$, aspergilosis invasiva $43 \%$ y candidiasis invasiva $28 \%$.

Otro estudio prospectivo de pacientes postrasplantados del grupo italiano trapianto midollo osseo entre los años 2008 y 2010 reporta una incidencia de mucormicosis de $3.7 \%$, aspergilosis invasiva $81.1 \%$ y candidemia $11 \%$.

En las guías de la tercera conferencia europea de infecciones (2013) para el diagnóstico y tratamiento de mucormicosis en pacientes con leucemia realizaron un estudio comparativo en 216 casos de aislamiento de mucorales sobre la actividad de anfotericina $\mathrm{B}$, posaconazol e itraconazol, en la tabla se muestra el que presenta mayor actividad (Tabla 1). ${ }^{22}$

\section{REFERENCIAS}

1. Kung HC, Huang PY, Chen WT, Ko BS, Chen YC, Chang SC, et al. 2016 guidelines for the use of antifungal agents in patients with invasive fungal diseases in Taiwan. $\mathrm{J}$ Microbiol Immunol Infect. 2018; 51 (1): 1-17.

2. Carrillo-Rodríguez VM, Soto-Ramos M, Hernández-Saldaña R, Hinojos-Gallardo LC, González-Ortiz S. Mucormicosis pulmonar en niños. Presentación de 3 casos y revisión de la literatura. Neumol Cir Torax. 2017; 76 (2): 96-101.

3. Imbernón A, Agud JL, Cuétara MS, Casqueiro JC, Nuñez $\mathrm{P}$, Domínguez AR et al. Successful therapy of progressive rhino-orbital mucormycosis caused by Rhizopus arrhizus with combined and sequential antifungal therapy, surgery and hyperbaric therapy. Med Mycol Case Rep. 2014; 6: 51-54.

4. Valdez-Geraldo CM, Zavala-Ruiz MG, Collado-Castro I, Ramírez-Villela C, Olguín A. Mucormicosis rinocerebral: Reporte de caso en escolar con cetoacidosis diabética. Rev Mex Neuroci. 2014; 15 (4): 229-233.

5. Danion F, Aguilar C, Catherinot E, Alanio A, DeWolf S, Lortholary $\mathrm{O}$ et al. Mucormycosis: new developments into a persistently devastating infection. Semin Respir Crit Care Med. 2015; 36 (5): 692-705.

6. Alemayehu T. Rhino-orbito-cerebral mucormycosis: neglected mycoses in childhood malignancies. Virol-mycol. 2017; 6 (2).

7. Bravo JH, Agudelo AM, Cortés A, Matta L. Mucormicosis rino-órbito-cerebral de origen dental. Biomédica. 2018; 38 (1): 27-31.

8. Mutchnick S, Soares D, Shkoukani M. To exenterate or not? An unusual case of pediatric rhinocerebral mucormycosis. Int J Pediatr Otorhinolaryngol. 2015; 79 (2): 267-270.

9. Pana ZD, Seidel D, Skiada A, Groll AH, Petrikkos G, Cornely $O A$ et al. Invasive mucormycosis in children: an epidemiologic study in European and non-European countries based on two registries. BMC Infect Dis. 2016; 16 (1): 667.

10. Jensen TSR, Arendrup MC, von Buchvald C, Frandsen TL, Juhler M, Nygaard U. Successful treatment of rhino-orbitalcerebral mucormycosis in a child with leukemia. J Pediatr Hematol Oncol. 2017; 39 (4): e211-e215. 
11. Tortorano AM, Richardson M, Roilides E, van Diepeningen A, Caira M, Munoz P et al. ESCMID and ECMM joint guidelines on diagnosis and management of hyalohyphomycosis: Fusarium spp., Scedosporium spp. and others. Clin Microbiol Infect. 2014; 20 Suppl 3: 27-46.

12. Asano-Mori $Y$. Diagnosis and treatment of mucormycosis in patients with hematological malignancies. Med Mycol J. 2016; 57 (4): J155-J162.

13. Millon L, Larosa F, Lepiller Q, Legrand F, Rocchi S, Daguindau E et al. Quantitative polymerase chain reaction detection of circulating DNA in serum for early diagnosis of mucormycosis in immunocompromised patients. Clin Infect Dis. 2013; 56 (10): e95-101.

14. Abela L, Toelle SP, Hackenberg A, Scheer I, Güngör T, Plecko B. Fatal outcome of rhino-orbital-cerebral mucormycosis due to bilateral internal carotid occlusion in a child after hematopoietic stem cell transplantation. Pediatr Infect Dis J. 2013; 32 (10): 1149-1150.

15. Chamdine O, Gaur AH, Broniscer A. Effective treatment of cerebral mucormycosis associated with brain surgery. Pediatr Infect Dis J. 2015; 34 (5): 542-543.

16. Chitasombat MN, Kontoyiannis DP. Treatment of mucormycosis in transplant patients: role of surgery and of old and new antifungal agents. Curr Opin Infect Dis. 2016; 29 (4): 340-345.

17. Drogari-Apiranthitou M, Mantopoulou FD, Skiada A, Kanioura L, Grammatikou M, Vrioni $G$ et al. In vitro antifungal susceptibility of filamentous fungi causing rare infections: synergy testing of amphotericin $\mathrm{B}$, posaconazole and anidulafungin in pairs. J Antimicrob Chemother. 2012; 67 (8): 1937-1940.

18. Almyroudis NG, Sutton DA, Fothergill AW, Rinaldi MG, Kusne S. In vitro susceptibilities of 217 clinical isolates of zygomycetes to conventional and new antifungal agents. Antimicrob Agents Chemother. 2007; 51 (7): 2587-290.

19. Vitale RG, de Hoog GS, Schwarz P, Dannaoui E, Deng S, Machouart $\mathrm{M}$ et al. Antifungal susceptibility and phylogeny of opportunistic members of the order mucorales. J Clin Microbiol. 2012; 50 (1): 66-75.

20. Pagano L, Cornely OA, Busca A, Caira M, Cesaro S, Gasbarrino $C$ et al. Combined antifungal approach for the treatment of invasive mucormycosis in patients with hematologic diseases: a report from the SEIFEM and FUNGISCOPE registries. Haematologica. 2013; 98 (10): e127-e130.

21. Kyvernitakis A, Torres HA, Jiang Y, Chamilos G, Lewis $\mathrm{RE}$, Kontoyiannis DP. Initial use of combination treatment does not impact survival of 106 patients with haematologic malignancies and mucormycosis: a propensity score analysis. Clin Microbiol Infect. 2016; 22 (9): 811.e1-811.e8.

22. Skiada A, Lanternier F, Groll AH, Pagano L, Zimmerli S, Herbrecht $R$ et al. Diagnosis and treatment of mucormycosis in patients with hematological malignancies: guidelines from the 3rd European Conference on Infections in Leukemia (ECIL 3). Haematologica. 2013; 98 (4): 492-504.

Correspondencia:

Gabriela Estefanía Valdés de la Torre Centro Médico Nacional 20 de Noviembre, Instituto de Seguridad y Servicios Sociales de los Trabajadores del Estado (ISSSTE).

Av. Félix Cuevas Núm. 540, Esq. Avenida Coyoacán, Col. Del Valle, 03229, Alcaldía Benito Juárez, CDMX. Teléfono: 520050 03, ext. 14327-8

E-mail: stephyvaldes@gmail.com 